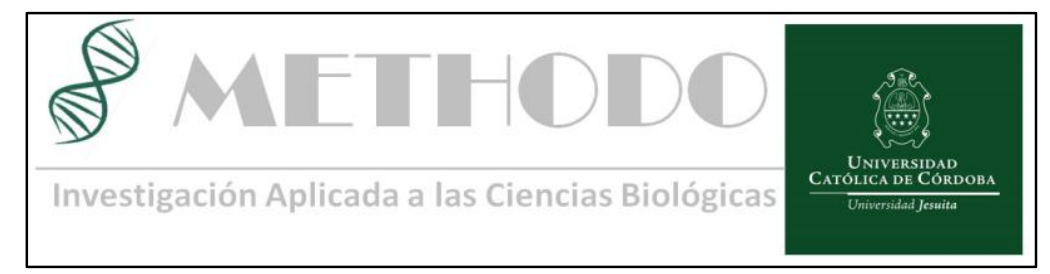

ARTICULO ORIGINAL Methodo 2019;4(1):12-21

DOI: $10.22529 / \mathrm{me} .2019 .4(1) 04$

\title{
Maduración pulmonar fetal: Efectos sobre la morbilidad respiratoria en prematuros tardíos
}

\section{Fetal pulmonary maturation: Effects on respiratory morbidity in late preterm infants}

\author{
Luciana Torres ${ }^{1}$, Esteban Ceballos ${ }^{2}$ \\ 1Servicio de Hospital Pedro Moguillansky - Cipolletti. Rio Negro. \\ 2 Servicio de Neonatología Clínica Universitaria Reina Fabiola. Universidad Católica de Córdoba \\ Correspondencia: Luciana Torres Servicio de Clínica Universitaria Reina Fabiola. Oncativo 1248 -X5004FHP- Córdoba, Argentina; e- \\ mail:lulitorres_12@hotmail.com.
}

\section{Resumen}

INTRODUCCIÓN: Los recién nacidos entre las 34 y las 36 semanas de gestación (SEG), definidos actualmente como "prematuros tardíos" (RNPTT) requieren en mayor frecuencia de internación en unidades de cuidados intensivos neonatales, siendo la morbilidad respiratoria una de las principales causas de internación ${ }^{1}$. El uso de corticoides prenatales ha demostrado mejorar la función pulmonar en prematuros extremos (<27,6 SEG), muy prematuros (28-31,6 SEG) y prematuros moderados, (32-36,6 SEG) pero no han sido evaluados en forma extensa en el período prematuro tardío y no existen datos concluyentes al respecto ${ }^{2}$.

OBJETIVO: Comparar la frecuencia en que se presenta la morbilidad respiratoria en RNPTT con o sin maduración pulmonar previa. Establecer los factores asociados a morbilidad respiratoria en RNPTT. Comparar los pacientes RNPTT con y sin maduración pulmonar: los requerimientos de internación en general, requerimiento de internación por morbilidad respiratoria y en estos, días de oxigenoterapia y necesidad de ARM y/o VNI.

MÉTODO: Diseño observacional, retrospectivo y analítico. Población constituida por todos los RNPTT que nacieron en la CURF entre el 1 de enero de 2017 y el 31 de diciembre de 2017. Las variables maternas y neonatales se compararán entre los dos grupos RNPTT con o sin maduración pulmonar. La frecuencia de presentación de la morbilidad respiratoria entre los RNPTT con o sin maduración pulmonar, los factores asociados a morbilidad respiratoria, los requerimientos de internación y necesidad de ARM y VNI se compararon con la prueba de chicuadrado siendo estadísticamente significativa la diferencia entre los mismos con $\mathrm{P} \leq 0,05$. Los días de internación se compararon mediante la prueba de $\mathrm{T}$ de Student. El Software que se utilizó para procesar los datos estadísticos será el SPSS versión 11.0 (Chicago, IL).

RESULTADOS: De los RNPTT estudiados (160) N=86 (53.7\%) recibieron corticoides prenatales completos. La edad gestacional media (DE) de administración fueron $35,2(2,2)$ semanas. La frecuencia de morbilidad respiratoria fue de $21,2 \%$. No hubo diferencia en la frecuencia de morbilidad respiratoria global entre ambos grupos ( 0 ,3777). El sexo masculino $(51,3 \%$ vs $49.0 \%$; $p=0,0034)$ la APP $(34,09 \% ; p=$ 0,0197 ) y morbilidad materna (preeclampsia, app, diabetes gestacional, corioamnionitis, RPM, placenta previa, HIE) $(70,65 \% ; \mathrm{p}=0,0062)$ se asociaron a morbilidad respiratoria. No hubo diferencias en el requerimiento de internación ni en el número de días de internación entre ambos grupos. Tampoco se observó diferencias en el requerimiento de oxígeno, ARM, VNI, uso de surfactante y necesidad de reanimación. Tampoco se hallaron diferencias en la frecuencia de enfermedad de membrana hialina, taquipnea transitoria del recién nacido, neumotórax. Hubo tendencia a una disminución de la frecuencia de 
SDRA $(6,35 \%$ vs $33.33 \%$, p= 0,051) en el grupo que recibió maduración pulmonar como así también una mayor frecuencia de apneas $25 \%(\mathrm{p}=0,0392)$.

CONCLUSIÓN: En este estudio la maduración pulmonar con corticoides no influencio la frecuencia de morbilidad respiratoria global. Sin embargo, al analizar las patologías respiratorias de manera individual, encontramos una tendencia a la disminución de SDRA en el grupo madurado. La frecuencia de apneas fue mayor en el grupo que recibió corticoides lo que requiere un análisis con mayor profundidad. El sexo masculino como así también la APP y morbilidad materna se asociaron a mayor frecuencia de morbilidad respiratoria.

Palabras claves: SDR, Neonatología, prematurez, oxigenoterapia, corticoides.

\section{Abstract}

INTRODUCTION: Newborns between 34 and 36 weeks of gestation (WG), currently defined as "latepreterm infants" (LPTI), require of admission in neonatal intensive care units more frequently, with respiratory morbidity being one of the main causes of hospitalization ${ }^{1}$. The use of prenatal corticosteroids has been shown to improve lung function in extremely preterm $(<27.6 \mathrm{WG})$, very preterm $(28-31.6 \mathrm{WG})$, and moderate preterm infants (32-36.6 WG), but it has not been evaluated extensively in the late-preterm period, leaving no conclusive data in this regard ${ }^{2}$.

OBJECTIVES: To compare the frequency of respiratory morbidity in LPTI with and without previous lung maturation. To establish the factors associated with respiratory morbidity in LPTI. - To compare LPTI patients with and without lung maturation: the requirements for hospitalization in general, the need for hospitalization due to respiratory morbidity, and in these cases, days of oxygen therapy and need for MRA and / or NIV.

METHOD: Observational, retrospective and analytical design. Population constituted by all LPTI that were born in the CURF between January 1, 2017 and December 31, 2017. Maternal and neonatal variables were compared between LPTI groups with and without lung maturation. The frequency of presentation of respiratory morbidity among LPTI with or without lung maturation, the factors associated with respiratory morbidity, the requirements for hospitalization and the need for MRA and NIV were compared with the chi-square test, considering statistically significant the difference between them with $\mathrm{P} \leq 0.05$. Days of hospitalization were compared using the Student's T test. The software used to process the statistical data was the SPSS version 11.0 (Chicago, IL).

RESULTS: Of the LPTI studied (160), $\mathrm{N}=86$ (53.7\%) received complete antenatal corticosteroids. Mean gestational age (SD) for administration was $35.2(2.2)$ weeks. The frequency of respiratory morbidity was $21.2 \%$. There was no difference in the frequency of overall respiratory morbidity between both groups ( $\mathrm{p}$ $0.3777)$. Male sex $(51,3 \%$ vs $49.0 \%$; $=0,0034)$, preterm birth risk $(34,09 \% ; p=0,0197)$ and maternal morbidity (preeclampsia, preterm birth risk, gestational diabetes, chorioamnionitis, preterm membrane rupture, previous placenta, pregnancy-induced hypertension) $(70,65 \% ; \mathrm{p}=0,0062)$ were associated to respiratory morbidity. There were no differences regarding hospitalization requirement or the number of hospitalization days between both groups. No differences were observed in oxygen requirement, MRA, NIV, use of surfactant and need for resuscitation. No differences were found in the frequency of hyaline membrane disease, newborn transient tachypnea and pneumothorax. There was a tendency to a decrease in the frequency of $\operatorname{RDS}(6.25 \%$ vs $33.33 \% ; \mathrm{p}=0.051)$, and a higher frequency of apneas $(25.0 \%$ vs $0 \%$; $\mathrm{p}=$ 0.0392 ) in the group of patients who received lung maturation.

CONCLUSION: In this study, pulmonary maturation with corticosteroids did not influence the frequency of global respiratory morbidity. However, when analyzing respiratory pathologies individually, we found a tendency to decrease of RDS in the matured group. The frequency of apneas was higher in the group that received corticosteroids, which requires further analysis. Male sex as well as preterm birth risk and maternal morbidity were associated to a higher frequency of respiratory morbidity.

Keywords: Neonatology, prematurity, oxygen therapy, corticosteroids. 


\section{Introducción}

Los recién nacidos entre las 34 y las 36 semanas de gestación (SEG), conocidos como "Recién Nacidos "prematuros tardíos" $(\text { RNPTT })^{1-3}$ requieren mayor frecuencia de internación en unidades de cuidados intensivos neonatales (UCIN), siendo la morbilidad respiratoria una de las principales causas ${ }^{4-6}$. El uso de corticoides prenatales ha demostrado mejorar la función pulmonar en prematuros extremos $(<27,6 \mathrm{sem})$, muy prematuros $(28-31,6 \mathrm{sem})$ y prematuros moderados, (3236,6 sem) pero no han sido evaluados en forma extensa en el período prematuro tardío y no existen datos concluyentes al respecto ${ }^{7-8}$ La tasa de nacimientos prematuros se ha incrementado en todo el mundo durante los últimos 10 años. Este aumento se debe, en parte, a la creciente tasa de nacimientos de RNPTT, los cuales representan al 70\% del total de los recién nacidos prematuros. Los RNPTT son una población de riesgo y presentan mayor morbimortalidad en comparación a los recién nacidos de termino ${ }^{4}$. Los RNPTT debido a su inmadurez presentan con mayor frecuencia trastornos adaptativos al nacer como Síndrome de dificultad respiratoria (SDR), Taquipnea transitoria, desregulación térmica, apneas, ictericia, dificultades en la alimentación e hipoglucemia ${ }^{1-5}$. El uso de corticoides prenatales ha demostrado ser beneficioso en mujeres con riesgo de parto prematuro antes de las 34 semanas, disminuyendo la morbimortalidad neonatal pero no ha sido evaluado en forma extensa en mujeres con amenaza de parto prematuro en el período prematuro tardío ${ }^{7-9}$. Desde hace más de 30 años se ha se han realizado estudios aleatorizados sobre maduración pulmonar fetal en embarazos menores a 32 Semanas de gestación, el trabajo más importante realizado al respecto fue el de Liggins y Howie que demostró que un solo curso de la terapia con corticoides prenatales administradas a mujeres con riesgo de parto prematuro redujo la incidencia y la gravedad del SDRA y la mortalidad en las de los recién nacidos prematuros ${ }^{9}$. Más de una docena de ensayos aleatorios han confirmado estos resultados. Los ensayos posteriores también demostrado que el tratamiento con corticoides prenatales mejora la estabilidad circulatoria en los recién nacidos prematuros, lo que resulta en menor incidencia de hemorragia intraventricular (HIV) y de enterocolitis necrotizante (NEC) en comparación con los recién nacidos prematuros no expuestos7-9. Los corticoides prenatales aceleran la maduración de los neumocitos tipo 1 y tipo 2 alveolares, dando lugar a cambios estructurales y bioquímicos que mejoran tanto la mecánica pulmonar (volumen y compliance pulmonar) y el intercambio de gases. La inducción madurativa de los neumocitos tipo 2 aumenta la producción de surfactante mediante la estimulación de la producción de las proteínas y las enzimas necesarias para la síntesis de fosfolípidos tensioactivos. Otros efectos de los corticoides prenatales incluyen la inducción de receptores beta pulmonares, los cuales juegan un papel en la liberación de surfactante y la eliminación del líquido alveolar mediante los canales de $\mathrm{Na}+$, hacia el intersticio alveolar. Para que estos cambios se produzcan, es necesario que los pulmones hayan alcanzado una etapa de desarrollo que sea biológicamente sensible a los corticoides. Los efectos a largo plazo han demostrado acción negativa en el crecimiento, menor talla, pero sin efecto en el crecimiento cerebral $y$ en el neurodesarrollo ${ }^{10-13}$

\section{Objetivos}

Comparar la frecuencia en que se presenta la morbilidad respiratoria en pacientes nacidos pretérmino tardío (RNPTT) con o sin maduración pulmonar previa.

Establecer las variables asociadas a morbilidad respiratoria en RNPTT. Comparar entre los pacientes RNPTT con y sin maduración pulmonar los requerimientos de internación en general, requerimiento de internación por morbilidad respiratoria y en estos, días de oxigenoterapia y necesidad de ARM y/o CPAP-VNI.

\section{Pacientes y métodos}

Diseño: Observacional, retrospectivo y analítico. Fuente de datos: Base de datos de los pacientes internados pertenecientes al Servicio de Neonatología y Tocoginecología de la Clínica Universitaria Reina Fabiola (CURF). Criterio de inclusión: Todos los pacientes prematuros tardíos definidos como los RN de 34 SEG y cero días postconcepcionales a 36 SEG y 6 días postconcepcionales, en adelante indicado con superíndice, 340 a 366, ingresados al Servicio de Tocoginecología y Neonatología de la CURF durante el periodo transcurrido del 1 de enero de 2017 y 31 de diciembre de 2017.

Grupo de estudio: Todos los RNPTT que recibieron corticoides prenatales. Dos dosis de betametasona separadas por $24 \mathrm{hs}$ una de la otra. Grupo control o de comparación: todos los RNPTT que NO recibieron maduración pulmonar con Betametasona previa a nacer.

Revista Methodo: Investigación Aplicada a las Ciencias Biológicas. Universidad Católica de Córdoba. Jacinto Ríos 571 Bo Gral. Paz. X5004FXS. Córdoba. Argentina. Tel.: (54) 3514517299 / Correo: methodo@ucc.edu.ar / Web: methodo.ucc.edu.ar | ARTICULO ORIGINAL Methodo 2019;4(1):12-21 
Criterios de Exclusión: Todo paciente prematuro tardío que padeció de una malformación o síndrome genético, todo paciente RNPTT que ingresó a neonatología derivado de otro centro médico. El motivo de este último criterio de exclusión se debe a que el traslado del paciente de un centro médico a otro aumenta la morbilidad general del paciente; aumentando de este modo el sesgo en la muestra de estudio.

\section{Variables a registrar}

a) Relacionadas a la madre: Edad: en años. Paridad: primípara, secundípara o multípara. Corticoides prenatales: no realizados, ciclo completo Morbilidad durante el embarazo: Hipertensión inducida por el embarazo. (HIE) Amenaza de parto prematuro (APP) Diabetes gestacional.(DBG) Ruptura prematura de membranas.(RPM) Corioamnionitis. Placenta Previa. Preeclampsia. (PE)

b) Relacionadas al neonato: Edad Gestacional: Estimada por fecha de última menstruación y/o ecografía precoz (11-14 sem) Género. Simple /gemelar Peso de nacimiento. Vía de nacimiento: Parto o Cesárea. Requerimiento de Reanimación.

c) Morbilidad respiratoria, nuestra variable de interés incluye: Enfermedad de Membrana Hialina $(\mathrm{EMH})$ o SDRA ${ }^{14}$ : Definido por evolución clínica, radiológica y días de requerimiento de oxigeno mayor a $48 \mathrm{hs}$ independientemente del requerimiento de Asistencia Respiratoria Mecánica (ARM)/surfactante. Clínicamente definido como síndrome de dificultad respiratoria caracterizado por taquipnea con frecuencias respiratorias mayores a 70 respiraciones por minuto, tiraje subcostal e intercostal, requerimiento de oxigeno suplementario con Fio2 mayor al $40 \%$ y presión positiva en la vía aérea. Secundario a déficit de surfactante endógeno e inadecuado lavado de líquido pulmonar alveolar. Hipertensión Pulmonar Persistente (HTPP)14: Definido por hipoxemia sostenida, labilidad a estímulos, requiriendo fio 2 mayor a $60 \%$ en forma sostenida y necesidad de vasodilatadores pulmonares para mantener oxigenación con o sin ecocardiograma doppler color que indique signos indirectos de HTPP y/o un gradiente a nivel de válvula tricúspide mayor a $19 \mathrm{cmH} 20$. Con o sin shunt de derecha a izquierda a través del foramen oval o ductus arterioso permeable. Taquipnea transitoria (TTRN)14: Definida como SDR leve que requiere un soporte de oxigenoterapia menor a las 48 hs. Con resolución completa del cuadro en dicho periodo de tiempo. Neumotorax (NTX)14: Fuga de aire a cavidad pleural diagnosticada por radiografía de torax con cambios clínicos acordes ha dicho evento.
Neumonía connatal14: Neumonía de origen perinatal inmediato secundaria a infección intrautero o canal de parto. Con epidemiologia materna. Con diagnostico mediante radiografía de torax y cambio clínico acorde. Que se desarrolla dentro de las 96 hs de vida.

Apneas14: Se define como cese del flujo respiratorio por más de 20 segundos con o sin bradicardia y acompañada de saturation de oxigeno por oximetría de pulso menor a $90 \%$, Cianosis y/o cambio del tono muscular.

d)Relacionadas a la terapéutica:

Necesidad de oxigenoterapia.

Necesidad de ARM.

Necesidad de uso de Surfactante.

Necesidad de CPAP nasal/ Ventilación No Invasiva (VNI).

Necesidad de internación y días

\section{Análisis estadístico}

La frecuencia de presentación de la respiratoria, los requerimientos de internación necesidad de ARM y VNI se compararon con la prueba de chicuadrado siendo estadísticamente significativa la diferencia entre los mismos con $\mathrm{P} \leq 0,05$. Los días de internación se compararon mediante prueba de T de Student. El Software que se utilizó para procesar los datos estadísticos fue el SPSS versión 11.0 (Chicago, IL).

\section{Aspectos éticos:}

Se garantizó la confidencialidad respecto de la identidad de los pacientes y sus datos filiatorios. Se respetó la ley 25326 de protección de datos personales y la ley 9496 de la Provincia de Córdoba. Todos los procedimientos estuvieron de acuerdo con lo estipulado en el reglamento de la ley general de salud en materia de investigación para la salud y se acoge al código ético establecido en la declaración de Helsinki de la asociación médica mundial. investigación sin riesgo, retrospectiva, no requiere consentimiento informado ni valoración por comité de ética institucional.

\section{Resultados}

En el periodo estudiado nacieron 2329 recién nacidos, de los cuales 2148 fueron a término y 192 pretermino. La tasa de prematuridad global anual de $8,2 \%$. De estos últimos 171 fueron RNPTT que corresponde a 7,34\% de los RN vivos y $89,06 \%$ de todos los prematuros. De este grupo 11 pacientes fueron excluidos del estudio debido a falta de información sobre maduración pulmonar y síndromes congénitos lo que representó una pérdida de 6,4\%. De los RNPTT, $102(63,7 \%)$ ingresaron a la unidad de neonatología. La 
frecuencia de morbilidad respiratoria fue del 21,2\%de la población seleccionada. La tasa de mortalidad para los RNPTT fue cero.

Las características demográficas, antecedentes obstétricos y perinatales y morbilidad respiratoria global en el periodo neonatal de los RNPTT incluidos en el estudio se muestran en la Tabla 1.

Los RNPTT estudiados (160) se dividieron en dos grupos según la administración prenatal de corticoides. Recibieron corticoides prenatales completos $\mathrm{N}=86(53.7 \%)$ a una edad gestacional media de 35,2 (2.2) semanas.

No hubo diferencia en la frecuencia de morbilidad respiratoria global entre ambos grupos $(p=0,377)$. Estos datos se observan en el Figura 1.

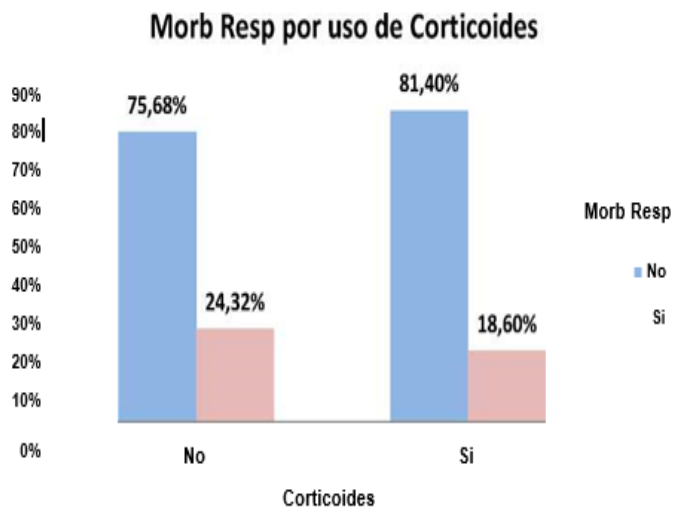

Figura 1. Frecuencia de morbilidad respiratoria en recién nacido pre término tardío según utilización de corticoides.

Se analizaron factores asociados a morbilidad respiratoria independientemente del uso de corticoides. El sexo masculino se asoció de manera estadísticamente significativa con morbilidad respiratoria $(p=0,0034)$. Las pacientes con amenaza de parto prematuro $(\mathrm{p}=0,0197)$ y morbilidad materna (preeclampsia, APP, diabetes gestacional, corioamnionitis, RPM, placenta previa, HIE $),(p=0,0062)$. presentaron mayor morbilidad respiratoria Hubo una tendencia al aumento de frecuencia de morbilidad respiratoria en pacientes añosas $\geq 35$ años $(p=0,0739)$. Estos datos se observan en Tabla 2

Por último, se comparó entre grupos la necesidad de internación, días de internación y patologías respiratorias además de la necesidad de técnicas de asistencia respiratoria. No hubo diferencias en el requerimiento de internación como así tampoco en los días de internación entre ambos grupos. Tampoco se hallaron diferencias en el requerimiento de oxígeno, ARM, VNI, uso de surfactante y necesidad de reanimación. Hubo una tendencia a la disminución en la frecuencia de SDRA $(p=0,0512)$ en el grupo que recibió maduración. En las otras patologías respiratorias, enfermedad de membrana hialina, neumotórax, taquipnea transitoria del recién nacido no se encontraron diferencias significativas entre ambos grupos. Se halló aumento de la frecuencia de apneas en el grupo que recibió maduración pulmonar de manera significativa $(\mathrm{p}=0,0392)$. Estos datos se observan en Tabla 3.

\section{Discusión}

Durante la última década son numerosas las investigaciones que confirman, de manera repetida, que los RNPTT presentan una morbilidad mayor que los nacidos a término debido a su inmadurez. ${ }^{2-3-5}$

A diferencia de la mayoría de la literatura publicada, en nuestro estudio no hubo diferencia significativa en la frecuencia de morbilidad respiratoria entre el grupo que recibio maduracion pulmonar y el que no la recibio. Sin embargo, al analizar las patologlas respiratorias de manera individual, encontramos una tendencia a la disminucion de la frecuencia de SDRA en el grupo madurado. Por el contrario, las apneas fueron mas frecuentes en el grupo que recibio maduracion y de manera significativa lo cual deberla ser estudiado con mayor profundidad.

Tambien se objetivo una influencia del sexo masculino en la morbilidad respiratoria.

En nuestra poblacion hubo un porcentaje de pacientes anosas > a 35 anos de $38 \%$. Se ha descripto que la edad materna avanzada se asocia con un mayor riesgo de complicaciones obstetricas, que en muchas ocasiones son la indication para la finalization de la gestation y tambien es mas frecuente la necesidad de tecnica de reproducción asistida que aumenta la probabilidad de embarazo multiple ${ }^{11}$.Se encontro una tendencia hacia el aumento de frecuencia de morbilidad respiratoria en este grupo de pacientes. Se ha descripto una relation directa con la via de finalización del embarazo, sin embargo, en nuestro estudio no se encontro diferencias entre la edad materna y via de finalizacion del mismo. Creemos conveniente poder estudiar una poblacion mas amplia y heterogenea. Al analizar algún trastorno materno (preeclampsia, APP, diabetes gestacional, corioamnionitis, RPM, placenta previa, HIE), se objetivo asociación con morbilidad respiratoria de manera significativa.

La frecuencia de APP fue del 28,3\%, a diferencia de otros trabajos ${ }^{2-10-13}$ observamos que influye directamente sobre la morbilidad respiratoria de manera estadísticamente significativa e independiente de la maduración pulmonar.

A la hora de decidir a qué edad gestacional se recomienda la administración prenatal de corticoides no solo influye la frecuencia de la

Revista Methodo: Investigación Aplicada a las Ciencias Biológicas. Universidad Católica de Córdoba. Jacinto Ríos 571 Bo Gral. Paz. X5004FXS. Córdoba. Argentina. Tel.: (54) 3514517299 / Correo: methodo@ucc.edu.ar / Web: methodo.ucc.edu.ar | ARTICULO ORIGINAL Methodo 2019;4(1):12-21 
enfermedad (los RNPTT presentan menos morbilidad respiratoria que los prematuros de una edad gestacional inferior), sino también el número total de pacientes que se verían beneficiados 9 .

Como ya hemos referido, los RNPTT constituyen el grupo más frecuente de prematuros, por lo que el estudio de la maduración pulmonar en dicha población tiene como objetivo poder reducir el número total de pacientes que ingresen a la unidad de neonatología lo que supone un menor coste socioeconómico. Sin embargo, en nuestro estudio no hubo diferencias en la necesidad de internación ni promedio de días de internación. Probablemente esto se deba al pequeño tamaño de la muestra.

Por otro lado, a pesar que nuestra población de pacientes madurados es mayor que la de no madurados y a la vez mayor que la de los demás estudios, la semana en que se realizó la maduración fue heterogénea, por lo que el efecto de los mismos podría reducirse e influir de manera directa en los resultados obtenidos.

Algunos estudios no apoyan la administración prenatal de corticoides. En el estudio de GyamfiBannerman et al, observacional y multicentrico, no encontraron que la exposición prenatal a los corticoides disminuyese la morbilidad respiratoria entre los $\mathrm{RNPTT}^{2}$. Una limitación importante de este estudio es que ninguna de las mujeres recibió los corticoides durante las 34-36 semanas y que se desconoce cuándo los recibieron, pues su efecto se reduce con el paso del tiempo.

Hasta el momento ningún ensayo clínico ha evaluado los efectos adversos a largo plazo de la administración de corticoides prenatales durante el periodo de la prematuridad tardía. Los más descriptos son la alteración del crecimiento y neurodesarrollo. En modelos animales se ha comprobado que los corticoides inducen la apoptosis y la muerte celular en el cerebro de los animales expuestos ${ }^{14}$. En la mayor la de los humanos el proceso de división neuronal ya se ha completado a las 24 semanas de gestación, no así el de los oligodendrocitos, principales células implicadas en las síntesis de mielina, cuyo crecimiento más rápido ocurre entre las 34 y 36 semanas de gestación ${ }^{1}$. Esto haría a los RNPTT más vulnerables a los posibles efectos neurológicos adversos de los corticoides prenatales que los más prematuros. Todo ello justifica la necesidad de un seguimiento a largo plazo de este gran grupo de prematuros y más estudios sobre los posibles efectos secundarios antes de confirmar su recomendación.

\section{Conclusión}

En este estudio la maduración pulmonar con corticoides no influencio la frecuencia de morbilidad respiratoria global, Sin embargo, al analizar las patologías respiratorias de manera individual, encontramos una tendencia a la disminución de SDRA en el grupo madurado. La frecuencia de apneas fue mayor en el grupo que recibió corticoides lo que requiere un análisis con mayor profundidad. El sexo masculino como así también la APP y la morbilidad materna se asociaron a mayor frecuencia de morbilidad respiratoria.

\section{Bibliografía}

1. Engle WA. A recommendation for the definition of «late preterm» (near-term) and the birth weight-gestational age classification system. Semin Perinatol. 2006;30(1):2-7.

2. Gyamfi-Bannerman C. Late Preterm Birth: Management Dilemmas. Obstetrics and Gynecology Clinics of North America. 2012;39(1):35-45.

3. Raju TNK, Higgins RD, Stark AR, Leveno KJ. Optimizing care and outcome for latepreterm (near-term) infants: a summary of the workshop sponsored by the National Institute of Child Health and Human Development. Pediatrics. 2006;118(3):1207-14.

4. Natarajan G, Shankaran S. Short- and LongTerm Outcomes of Moderate and Late Preterm Infants. Am J Perinatol. 2016;33(3):305-17.

5. Mahoney AD, Jain L. Respiratory Disorders

in Moderately Preterm, Late Preterm, and Early Term Infants. Clinics in Perinatology. 2013;40(4):665-78.

6. Clark RH. The epidemiology of respiratory failure in neonates born at an estimated gestational age of 34 weeks or more. J Perinatol. 2005;25(4):251-7.

7. ACOG Committee ON Obstetric Practice. ACOG Committee opinion, no 475.Antenatalcorticosteroid therapy for fetal maturation. Obstet Gynecol. 2011; 117:422-4.

8. Liggins GC, Howie RN. A controlled trial of antepartum glucocorticoid treatment for prevention of the respiratory distress syndrome in premature infants. Pediatrics. 1972;50(4):515-25.

9. Porto AMF, Coutinho IC, Correia JB, Amorim MMR. Effectiveness of antenatal corticosteroids in reducing respiratory 
disorders in late preterm infants: randomised clinical trial. BMJ. 2011;342:1696.

10. Gazquez Serrano IM, Arroyos Plana A, Diaz Morales O, Herraiz Perea C, Holgueras Bragado A. [Antenatal corticosteroid therapy and late preterm infant morbidity and mortality]. An Pediatr (Barc). 2014;81(6):374-82.

11. Ventolini G, Neiger R, Mathews L, Adragna $\mathrm{N}$, Belcastro $\mathrm{M}$. Incidence of respiratory disorders in neonates born between 34 and 36 weeks of gestation following exposure to antenatal corticosteroids between 24 and 34 weeks of gestation. Am J Perinatol. 2008;25(2):79-83.

12. Gyamfi-Bannerman C, Thom EA, Blackwell SC, Tita ATN, Reddy UM, Saade GR, et al. Antenatal Betamethasone for Women at Risk for Late Preterm Delivery. N Engl J Med. 2016; 80:230:8.

13. Whitelaw A, Thoresen M.Antenatal steroids and the developing brain.Arch Dis Child Fetal Neonatal Ed. 2000; 83:154-7.

14. Judith U. Hibbard. Consortium on Safe Labor, Respiratory morbidity in late preterm births. JAMA. 2010 28;304(4):419-25.

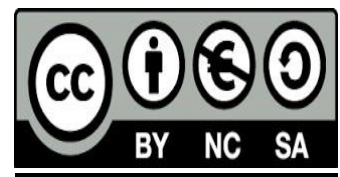




\section{Anexo tablas}

Tabla 1 Características generales de la población estudiada. CIR: crecimiento intrauterino restringido, HIE hipertensión inducida por el embarazo, APP amenaza de parto prematuro, RPM ruptura prematura de membranas, EMH enfermedad de membrana hialina, SDRA smdrome de distress respiratorio, ARM asistencia respiratoria mecánica, VNI ventilación no invasiva.

\begin{tabular}{|c|c|c|}
\hline & Frecuencia & Porcentaje (\%) \\
\hline \multicolumn{3}{|l|}{ Caracteristicas generales } \\
\hline \multicolumn{3}{|l|}{ Edad gestacional } \\
\hline 34 semanas & 42 & 26,25 \\
\hline 35 semanas & 39 & 24,38 \\
\hline 36 semanas & 79 & 49,38 \\
\hline Peso al nacer (media -DE) en gr & $2.941( \pm 67)$ & \\
\hline Sexo femenino & 78 & 48,75 \\
\hline Sexo masculino & 82 & 51,25 \\
\hline \multicolumn{3}{|l|}{ Datos de la gestacion } \\
\hline Edad materna $>35$ anos & 54 & 38 \\
\hline Edad materna (media-DE) en anos & $32,61( \pm 0,89)$ & \\
\hline Nullpara & 1 & 2,94 \\
\hline Primlpara & 17 & 50 \\
\hline Secundipara & 12 & 35,29 \\
\hline Multipara & 4 & 11,76 \\
\hline HIE & 8 & 5,16 \\
\hline Diabetes & 7 & 4,52 \\
\hline Primiparas & 73 & $\overline{47,4}$ \\
\hline Gemelar & 38 & 23,75 \\
\hline Preeclamsia & 12 & $\mathbf{7 , 7 4}$ \\
\hline CIR & 12 & 7,5 \\
\hline APP & 44 & 28,39 \\
\hline RPM & 14 & 9,03 \\
\hline \multicolumn{3}{|l|}{ Datos del parto } \\
\hline \multicolumn{3}{|l|}{ Tipo } \\
\hline Vaginal & 54 & 35,06 \\
\hline Cesarea & 100 & 64,9 \\
\hline Necesidad de reanimación & 22 & 13,75 \\
\hline \multicolumn{3}{|l|}{ Morbilidad global } \\
\hline Morbilidad Respiratoria & 34 & 21,25 \\
\hline Total & 160 & 100 \\
\hline EMH & 3 & 1,88 \\
\hline Taquipnea Transitoria & 15 & 9,38 \\
\hline SDR & 7 & 4,38 \\
\hline Apneas & 4 & 2,5 \\
\hline Oxigeno & 16 & 10 \\
\hline ARM & 4 & 2,5 \\
\hline $\begin{array}{ll}\text { VNI } \\
\end{array}$ & 3 & 1,88 \\
\hline
\end{tabular}

Revista Methodo: Investigación Aplicada a las Ciencias Biológicas. Universidad Católica de Córdoba. Jacinto Ríos 571 Bo Gral. Paz. X5004FXS. Córdoba. Argentina. Tel.: (54) 3514517299 / Correo: methodo@ucc.edu.ar / Web: methodo.ucc.edu.ar | ARTICULO ORIGINAL Methodo 2019;4(1):12-21 
Tabla 2 Análisis de factores de riesgo para morbilidad respiratoria. CIR: crecimiento intrauterino restringido, HIE: hipertensión inducida por el embarazo, APP: amenaza de parto prematuro, RPM: ruptura prematura de membranas.

\begin{tabular}{|c|c|c|c|}
\hline & \multicolumn{2}{|c|}{ Morbilidad respiratoria } & \multirow[t]{2}{*}{ Valor de $\mathbf{P}$} \\
\hline & SI & NO & \\
\hline \multicolumn{4}{|l|}{ Variable N (\%) } \\
\hline \multicolumn{4}{|l|}{ Trastorno embarazo } \\
\hline Ninguno & $57(89,06)$ & $7(10,94)$ & \\
\hline Preeclampsia & $8(66,67)$ & $4(33,33)$ & \\
\hline APP & $29(65,91)$ & $15(34,09)$ & 0,0197 \\
\hline DBT & $7(100)$ & $0(0)$ & \\
\hline Corioam & $2(66,67)$ & $1(33,33)$ & \\
\hline RPM & $10(71,43)$ & $4(28,57)$ & \\
\hline Placenta Previa & $2(50)$ & $2(50)$ & \\
\hline HIE & $7(87,5)$ & $1(12,5)$ & \\
\hline \begin{tabular}{|l|} 
Algún trastorno \\
\end{tabular} & $65(70,65)$ & $27(29,35)$ & 0,0062 \\
\hline \multicolumn{4}{|l|}{ Simple/Gemelar } \\
\hline Simple & $30(24,5)$ & $92(75,4)$ & \\
\hline Gemelar & $4(10,5)$ & $34(89,4)$ & 0,0642 \\
\hline \multicolumn{4}{|l|}{ Factores de riesgo } \\
\hline Edad materna $>35$ & $17(28,81)$ & $17(16,83)$ & 0,0739 \\
\hline CIR & $10(83,33)$ & $2(16,67)$ & \\
\hline \multicolumn{4}{|l|}{ Sexo } \\
\hline Femenino & $69(88,46)$ & $9(11,54)$ & \\
\hline Masculino & $57(69,51)$ & $25(30,49)$ & 0,0034 \\
\hline \multicolumn{4}{|l|}{ Terminación del } \\
\hline \begin{tabular}{|c|} 
Vaginal \\
\end{tabular} & $45(83,33)$ & $9(16,67)$ & \\
\hline Cesárea & $76(75,25)$ & $25(24,75)$ & \\
\hline
\end{tabular}


Tabla 3. Análisis de frecuencia de patologías respiratorias y necesidad de intervención e internación en recién nacido pre terminó tardío según maduración pulmonar. CIR: crecimiento intrauterino restringido, HIE: hipertensión inducida por el embarazo, APP: amenaza de parto prematuro, RPM: ruptura prematura de membranas, EMH: enfermedad de membrana hialina, SDRA: síndrome de distress respiratorio, ARM: asistencia respiratoria mecánica, VNI: ventilación no invasiva.

\begin{tabular}{|c|c|c|c|}
\hline \multirow[b]{2}{*}{ Variable } & \multicolumn{2}{|c|}{ Corticoides } & \multirow[b]{2}{*}{ Valor de $\mathbf{p}$} \\
\hline & $\mathbf{S i}$ & No & \\
\hline \multicolumn{4}{|l|}{ Patología respiratoria } \\
\hline EMH & $1(6,25)$ & $2(11,11)$ & 1,0000 \\
\hline Taquipnea Transitoria & $9(56,25)$ & $6(33,33)$ & 0,1792 \\
\hline SDRA & $1(6,25)$ & $6(33,33)$ & 0,0512 \\
\hline NTX & $0(0)$ & $1(5,56)$ & 1,0000 \\
\hline Apneas & $4(25)$ & $0(0)$ & 0,0392 \\
\hline \multicolumn{4}{|l|}{ Intervención } \\
\hline Oxígeno & $8(50)$ & $3(16,6)$ & 0,746 \\
\hline Reanimación & $9(56,25)$ & $13(72,22)$ & 0,3307 \\
\hline ARM & $1(6,25)$ & $3(16,67)$ & 0,6041 \\
\hline VNI & $1(6,25)$ & $2(11,11)$ & 1 \\
\hline Surfactante & $1(6,25)$ & $3(16,67)$ & 0,6041 \\
\hline \multicolumn{4}{|l|}{ Internación } \\
\hline Necesidad de internación & $15(93,75)$ & $15(83,33)$ & 0,6041 \\
\hline Dias de internación & 9,69 & 6,5 & 0,0715 \\
\hline
\end{tabular}

\section{(1)(0) \\ BY NC SA}

